\title{
Hepatitis B- and C-related hepatocellular carcinomas yield different clinical features and prognosis
}

\author{
Chien-Hung Chen ${ }^{a}$, Guan-Tarn Huang ${ }^{a}$, Pei-Ming Yang ${ }^{a}$, Pei-Jer Chen ${ }^{a, b, c}$, \\ Ming-Yang Lai ${ }^{a, b}$, Ding-Shinn Chen ${ }^{a, b, c}$, Jung-Der Wang ${ }^{a, d, e}$, Jin-Chuan Sheu ${ }^{a, *}$ \\ ${ }^{a}$ Department of Internal Medicine, National Taiwan University Hospital and National Taiwan University College of Medicine, \\ 7 Chung-Shan South Road, Taipei, Taiwan \\ ${ }^{\mathrm{b}}$ Graduate Institute of Clinical Medicine, National Taiwan University Hospital and National Taiwan University College of Medicine, \\ Taipei, Taiwan \\ ${ }^{c}$ Hepatitis Research Center, National Taiwan University Hospital and National Taiwan University College of Medicine, Taipei, Taiwan \\ ${ }^{\mathrm{d} C e n t e r ~ f o r ~ H e a l t h ~ R i s k ~ A s s e s s m e n t ~ a n d ~ P o l i c y, ~ C o l l e g e ~ o f ~ P u b l i c ~ H e a l t h, ~ N a t i o n a l ~ T a i w a n ~ U n i v e r s i t y, ~ T a i p e i, ~ T a i w a n ~}$ \\ ${ }^{\mathrm{e}}$ Institute of Occupational Medicine and Industrial Hygiene, College of Public Health, National Taiwan University, Taipei, Taiwan
}

\section{A R T I C L E I N F O}

\section{Article history:}

Received 9 May 2006

Accepted 12 June 2006

Available online 22 August 2006

Keywords:

Hepatocellular carcinoma

Hepatitis B virus

Hepatitis $C$ virus

Survival

\begin{abstract}
A B S T R A C T
The purpose of this study was to compare the clinical features and survival in patients with hepatitis B virus-related hepatocellular carcinoma (HBV-HCC) and hepatitis C virus-related hepatocellular carcinoma (HCV-HCC). A total of 2820 HCC patients were included. The mean age of HBV-HCC patients was $52.5 \pm 13.4$ years, while it was $64.8 \pm 8.7$ years in HCV-HCC patients. The male/female ratio was 7.8 in HBV-HCC, while it was 1.7 in HCVHCC. The mean $\alpha$-fetoprotein level in HBV-HCC was $11,661 \pm 22,805 \mathrm{ng} / \mathrm{mL}$, while it was $5079 \pm 15,005 \mathrm{ng} / \mathrm{mL}$ in HCV-HCC. The mean tumour size was $6.4 \pm 4.1 \mathrm{~cm}$ in HBV-HCC, while it was $4.6 \pm 3.1 \mathrm{~cm}$ in HCV-HCC. The median survival was 11.1 months in HBV-HCC, while it was 23.9 months in HCV-HCC. Compared with HBV-HCC patients, HCV-HCC patients were older, had a lower male/female ratio, lower white blood cell count, lower serum albumin level, higher serum ALT level, lower serum $\alpha$-fetoprotein level, smaller tumour size and survived longer.
\end{abstract}

(c) 2006 Elsevier Ltd. All rights reserved.

\section{Introduction}

Hepatocellular carcinoma (HCC) is one of the most common malignancies in the world, especially in sub-Saharan Africa and Southeast Asia. Since 1984, it has been the leading cause of cancer death in Taiwan. ${ }^{1}$ Around 7000 people die of HCC annually and around 8000 new HCC cases are found every year in Taiwan. ${ }^{2}$ The high risk groups of HCC in Taiwan include patients chronically infected with hepatitis $B$ virus (HBV) or hepatitis C virus (HCV), liver cirrhosis, or family history of HCC. Among them, HBV and HCV chronic infections are the two major aetiologies for HCCs in Taiwan. ${ }^{3}$
Several studies have examined the differences among HBVrelated HCC (HBV-HCC) and HCV-related HCC (HCV-HCC). ${ }^{4-17}$ However, there were still some limitations in the previous studies. Most investigations focused on surgical patients only. ${ }^{4-6,8-10,13-15}$ The surgical patients represented the better prognosis groups out of all HCC patients. Thus, the results might not be generalised to the whole HBV-HCC and HCVHCC patients. Furthermore, the case numbers were small and the follow-up period was not long enough. Only a few studies explored HCC patients receiving different treatment modalities. ${ }^{7,11,12}$ However, these studies were from the areas where the HCV was the predominant aetiology for HCC.

* Corresponding author: Tel.: +886 2 23123456x7266; fax: +886 223819723.

E-mail address: sheuhcc@ntumc.org (J.-C. Sheu).

0959-8049/\$ - see front matter (c) 2006 Elsevier Ltd. All rights reserved.

doi:10.1016/j.ejca.2006.06.007 
Whether such conclusions can also be applied in the HBV-endemic area, like Taiwan, is still unclear. Thus, the purpose of this study is to compare the clinical features and survival in patients with HBV-HCC and HCV-HCC in Taiwan.

\section{Patients and methods}

\subsection{Patients and data collection}

The medical records with the diagnosis of HCC in National Taiwan University Hospital from 1981 to 2000 June were retrospectively reviewed. Those who received their initial treatments for HCC at other hospitals were excluded. The diagnosis of HCC was based on either histologic examination of tumour tissues or typical radiological image pictures. The following data were collected at diagnosis: name, chart number, national citizen identification number, sex, birthday, date of HCC diagnosis, date of death and date of last follow-up, diagnostic criteria for HCC, modality of first treatment, presence of liver cirrhosis, Child-Pugh stage and its constitutive variables (albumin, bilirubin, prothrombin time, ascites and encephalopathy), hepatitis B surface antigen (HBsAg) and antibody for hepatitis $\mathrm{C}$ virus (anti-HCV) status, WBC, haemoglobin, platelet, AST, ALT, alkaline phosphatase (ALP), BUN, creatinine, serum $\alpha$-fetoprotein (AFP), tumour size, tumour number, evidence of portal vein invasion, bile duct invasion or distant metastases. Only patients with both HBsAg and anti-HCV available data were enrolled in this study.

\subsection{Aetiology of HCC}

HBV-HCC was defined as HCC with a positive HBsAg status and a negative anti-HCV status. HCV-HCC was defined as HCC with a negative HBsAg status and a positive anti-HCV status. The HBV plus HCV-related HCC (HBV+HCV-HCC) was defined as HCC with a positive status for both HBsAg and anti-HCV. The non-HBV and non-HCV-related HCC (NBNCHCC) was defined as HCC with a negative status for both HBsAg and anti-HCV.

\subsection{Searching for fatalities}

To verify patients' deaths, we used the national citizen identification (ID) number of patients with HCC to search the mortality data bank established by the Statistics Office, Department of Health, Taiwan. The mortality data bank includes data from the certificate of death, which contains the demographic data of the patient; the time, place, and cause of death; and the name of the person who issued the document. The mortality data bank included patients who had expired before 31st December 2001. Thus, cases that still survived after 31st December 2001 were right censored. Since the mortality data bank did not contain the original ID number before 1985, a verification and re-insertion of ID was completed using the database of Internal Revenue Service. ${ }^{18}$

\subsection{Statistical analysis}

Statistical analysis of differences among the four groups was done using Pearson's $\chi^{2}$ test. Survival curves were estimated using the Kaplan-Meier method and the differences in the survival rates between the groups were compared by the log-rank test. All statistical analyses were performed using the SAS software, Version 8 (Statistical Analysis System, Cary, NC, United States). The statistical significance was defined as $p<0.05$.

\section{Results}

A total of 4968 Taiwanese HCC patients were collected. Medical records in which details pertaining to any of the following three items, HBsAg, anti-HCV and initial treatment method, were missing were excluded. Most of the excluded records were due to missing anti-HCV data. Thus, a total of 2820 patients were analysed in this study, including 1698 HBV-HCC (60.2\%), 613 HCV-HCC (21.7\%), 214 HBV+HCV-HCC (7.6\%) and 295 NBNC-HCC (10.5\%).

\subsection{Age and sex of HCC patients}

As shown in Table 1, the mean age of HCV-HCC patients was 4 years older than those of HBV+HCV-HCC or NBNC-HCC patients, and 12 years older than that of HBV-HCC patients. The male/female ratio (7.8) was highest in HBV-HCC, while it was lowest in HCV-HCC (1.7).

\subsection{Biochemistry in HCC patients}

The HCV-HCC patients had lower WBC and platelet counts. The serum albumin levels were lower in the HCV-HCC and HBV+HCV-HCC patients compared with those in the HBVHCC or NBNC-HCC patients. The mean ALT level was lowest in the NBNC-HCC patients. Though the ALT level was slightly higher in the HCV-HCC patients, there was no statistically significant difference in the mean ALT level between HBV-HCC and HCV-HCC patients. The mean bilirubin levels, prolongation of prothrombin time, presence of ascites or encephalopathy were similar among all four groups.

\subsection{Tumour characteristics and staging of HCCs}

The HBV-HCC group had higher AFP levels than other groups. The mean tumour size was largest in the NBNC-HCC group, followed by the HBV-HCC, HBV+HCV-HCC and HCV-HCC groups $\quad(7.6 \pm 4.5 \mathrm{~cm}, \quad 6.4 \pm 4.1 \mathrm{~cm}, \quad 5.7 \pm 3.8 \mathrm{~cm}$, and $4.6 \pm 3.1 \mathrm{~cm}$, respectively). There were no statistically significant differences in the tumour numbers between different groups. The HCV-HCC group had a higher percentage of patients in the earlier stages, while the NBNC-HCC group had the lowest percentage of patients in the earlier stage. The resectable rate was similar among all four groups $(42.3 \%$ in HBV-HCC, $40.5 \%$ in HCV-HCC, $40.7 \%$ in HBV+HCV-HCC, and $44.1 \%$ in NBNC-HCC) (Fig. 1).

\subsection{Survival}

As shown in Table 2 and Fig. 2, the patients with HCV-HCC had the best overall survival. However, it was interesting to find that the better survival of HCV-HCC patients happened in the initial 5 years, while the survival of HCV-HCC patients 


\begin{tabular}{|c|c|c|c|c|c|}
\hline Aetiology & $\operatorname{HBV}(N=1698)$ & $\operatorname{HCV}(N=613)$ & $\mathrm{HBV}+\mathrm{HCV}(\mathrm{N}=214)$ & NBNC $(N=295)$ & $p$ \\
\hline Age (years) & $52.5 \pm 13.4$ & $64.8 \pm 8.7$ & $60.7 \pm 10.4$ & $60.4 \pm 14.5$ & $<0.0001$ \\
\hline Male/female (ratio) & $1505 / 193(7.8)$ & $384 / 229(1.7)$ & $161 / 53(3.0)$ & $218 / 77(2.8)$ & $<0.0001$ \\
\hline White blood cell $(/ \mu \mathrm{L})$ & $6473 \pm 3064$ & $5639 \pm 3318$ & $6008 \pm 2832$ & $7656 \pm 4772$ & $<0.0001$ \\
\hline Haemoglobin (g/dL) & $12.9 \pm 2.3$ & $12.2 \pm 2.1$ & $12.3 \pm 2.2$ & $12.2 \pm 2.3$ & $<0.0001$ \\
\hline Platelet $(\mathrm{K} / \mu \mathrm{L})$ & $188 \pm 113.7$ & $128 \pm 77.6$ & $158 \pm 113.3$ & $226 \pm 109.8$ & $<0.0001$ \\
\hline PT prolonged (s) & $2.0 \pm 2.5$ & $2.1 \pm 2.1$ & $2.0 \pm 2.5$ & $1.6 \pm 2.5$ & 0.094 \\
\hline Albumin $(g / d L)$ & $3.6 \pm 0.6$ & $3.5 \pm 0.6$ & $3.5 \pm 0.5$ & $3.6 \pm 0.6$ & $<0.0001$ \\
\hline Bilirubin $(\mathrm{mg} / \mathrm{dL})$ & $2.0 \pm 3.9$ & $1.7 \pm 3.0$ & $2.0 \pm 4.4$ & $2.4 \pm 4.6$ & 0.128 \\
\hline Ascites (+/-) & $302 / 1287$ & $95 / 482$ & $41 / 164$ & $51 / 220$ & 0.536 \\
\hline Encephalopathy(+/-) & $10 / 1627$ & $5 / 585$ & $2 / 207$ & $2 / 281$ & 0.903 \\
\hline $\operatorname{ALT}(\mathrm{U} / \mathrm{L})$ & $65.8 \pm 100.8$ & $75.9 \pm 79.5$ & $69.8 \pm 53.0$ & $44.9 \pm 46.7$ & $<0.0001$ \\
\hline Child-Pugh stage (A/B/C) & $1097 / 248 / 46$ & $401 / 96 / 11$ & $138 / 31 / 3$ & $180 / 38 / 9$ & 0.716 \\
\hline $\operatorname{AFP}(\mathrm{ng} / \mathrm{mL})$ & $11,661 \pm 22,805$ & $5079 \pm 15,005$ & $5852 \pm 15,966$ & $7639 \pm 18,603$ & $<0.0001$ \\
\hline Tumour number $(1 / 2 / 3 / \geqslant 4)$ & $970 / 251 / 101 / 197$ & $363 / 93 / 44 / 83$ & $128 / 34 / 14 / 16$ & $172 / 41 / 15 / 26$ & 0.585 \\
\hline Mean tumour size $(\mathrm{cm})$ & $6.4 \pm 4.1$ & $4.6 \pm 3.1$ & $5.7 \pm 3.8$ & $7.6 \pm 4.5$ & $<0.0001$ \\
\hline CLIP stage $0 / 1 / 2 / 3 / 4-6$ & $258 / 343 / 266 / 129 / 77$ & $144 / 157 / 96 / 37 / 13$ & $37 / 52 / 35 / 15 / 4$ & $57 / 39 / 37 / 22 / 10$ & $<0.0001$ \\
\hline
\end{tabular}

HBV, hepatitis B virus; HCV, hepatitis C virus; PT, prothrombin time.

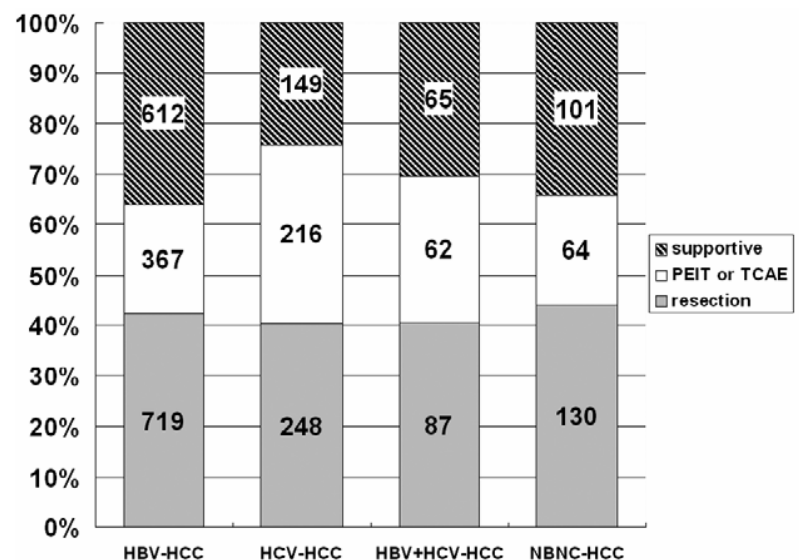

Fig. 1 - Distributions of treatment modalities in HCCs. The numbers within the bar indicate case numbers. TACE, transarterial chemoembolisation; PEIT, percutaneous ethanol injection therapy.

Table 2 - Cumulative survival rates and median survival of the 2820 HCC patients as a whole

\begin{tabular}{lllcl} 
Aetiology & HBV & HCV & HBV+HCV & BNC \\
\hline Median survival (months) & 11.1 & 23.9 & 14.0 & 15.0 \\
1 year & $48.6 \%$ & $64.6 \%$ & $54.6 \%$ & $52.6 \%$ \\
3 year & $28.3 \%$ & $40.8 \%$ & $32.9 \%$ & $33.6 \%$ \\
5 year & $21.0 \%$ & $23.1 \%$ & $19.1 \%$ & $24.3 \%$ \\
10 year & $12.8 \%$ & $10.5 \%$ & $7.2 \%$ & $17.5 \%$ \\
\hline
\end{tabular}

was worse than NBNC-HCC patients at 10 years. In patients who received resection as the initial treatment, though the survival was better in the NBNC-HCC groups in the 10 years, there were no statistically significant differences between different groups (Table 3, Fig. 3). There were no statistically significant differences in the disease-free survival between different groups in surgical patients. In patients who received transarterial chemoembolisation (TACE) or percutaneous eth-

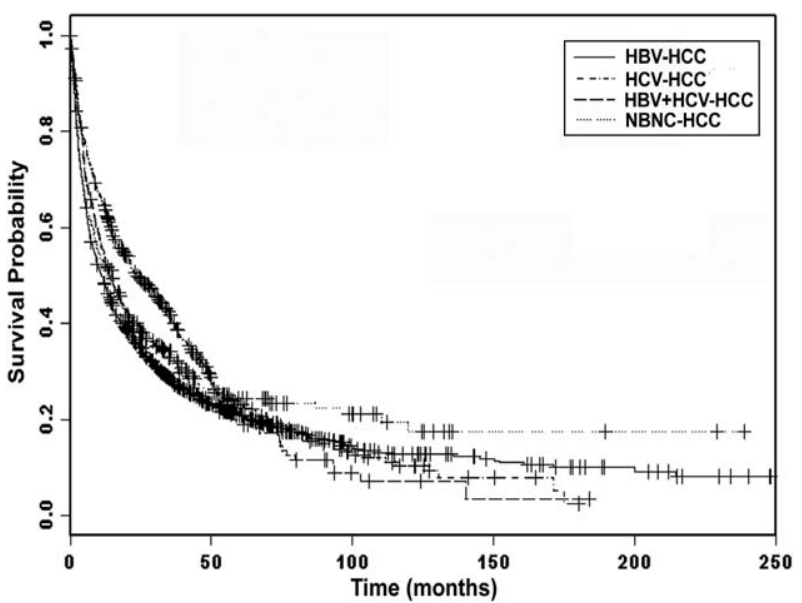

Fig. 2 - Kaplan-Meier estimated survival curves of HCC patients as a whole. The HCV-HCC group showed a better survival than the HBV-HCC $(p<0.05)$ or NBNC-HCC $(p<0.05)$ groups. HBV-HCC, HBV-related HCC; HCV-HCC, HCV-related HCC; HBV+HCV-HCC, HBV plus HCV-related HCC, NBNCHCC: non-HBV-non-HCV-related HCC.

Table 3 - Cumulative survival rates and median survival of the 1184 HCC patients who received surgical resection as the initial treatment

\begin{tabular}{lllcl} 
Aetiology & HBV & HCV & HBV+HCV & NBNC \\
\hline Median survival (months) & 44.2 & 50.6 & 46.2 & 53.2 \\
1 year & $77.0 \%$ & $85.0 \%$ & $78.1 \%$ & $80.8 \%$ \\
3 year & $54.2 \%$ & $65.3 \%$ & $58.8 \%$ & $63.8 \%$ \\
5 year & $42.7 \%$ & $44.7 \%$ & $40.7 \%$ & $48.3 \%$ \\
10 year & $27.2 \%$ & $21.8 \%$ & $17.7 \%$ & $37.7 \%$ \\
\hline
\end{tabular}

anol injection therapy (PEIT) as the initial treatment, the HCVHCC patients had the best survival. However, the advantage of better survival in HCV-HCC patients disappeared after 5 years (Table 4, Fig. 4). In patients who received supportive care or 


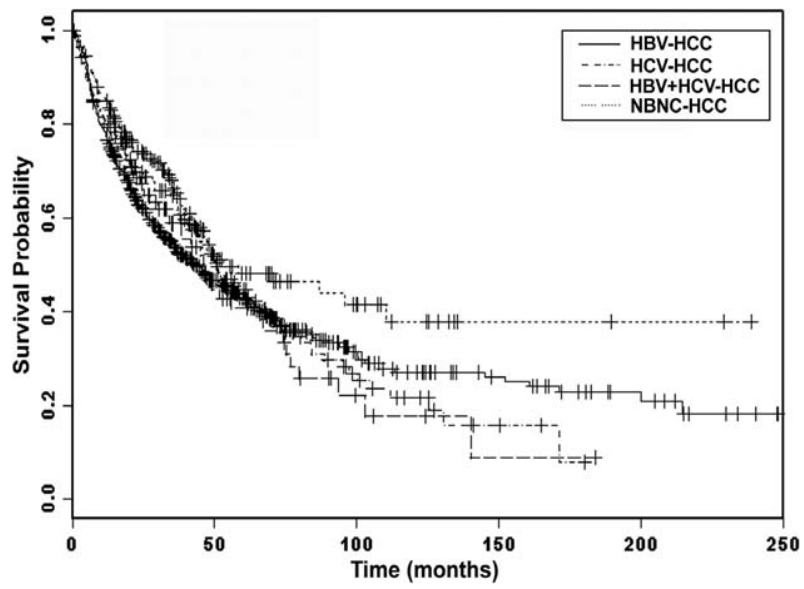

Fig. 3 - Kaplan-Meier estimated survival curves of HCC patients underwent surgical resection. There were no statistically significant differences in the survival among groups ( $p=0.188)$. HBV-HCC, HBV-related HCC; HCV-HCC, HCV-related HCC; HBV+HCV-HCC, HBV plus HCV-related HCC, NBNC-HCC: non-HBV-non-HCV-related HCC.

Table 4 - Cumulative survival rates of the 709 HCC patients who received TACE or PEIT as the initial treatment

\begin{tabular}{lllcl} 
Aetiology & HBV & HCV & HBV+HCV & NBNC \\
\hline Median survival (months) & 12.6 & 22.8 & 16.7 & 15.0 \\
1 year & $53.0 \%$ & $70.6 \%$ & $67.7 \%$ & $58.4 \%$ \\
3 year & $19.5 \%$ & $35.2 \%$ & $28.4 \%$ & $19.0 \%$ \\
5 year & $12.1 \%$ & $12.3 \%$ & $9.0 \%$ & $11.4 \%$ \\
10 year & $3.7 \%$ & $3.1 \%$ & $3.0 \%$ & $0 \%$ \\
\hline
\end{tabular}

TACE, transarterial chemoembolisation; PEIT, percutaneous ethanol injection therapy.

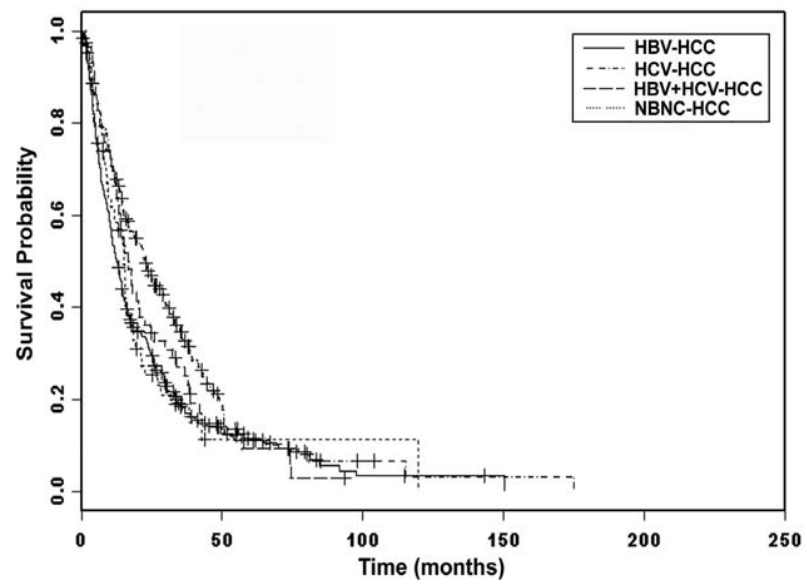

Fig. 4 - Kaplan-Meier estimated survival curves of HCC patients who underwent TACE or PEIT. The HCV-HCC group showed a better survival than the HBV-HCC $(p<0.001)$ or NBNC-HCC $(p<0.05)$ groups. HBV-HCC, HBV-related HCC; HCV-HCC, HCV-related HCC; HBV+HCV-HCC, HBV plus HCVrelated HCC, NBNC-HCC: non-HBV-non-HCV-related HCC. TACE, transarterial chemoembolisation; PEIT, percutaneous ethanol injection therapy.
Table 5 - Cumulative survival rates of the 927 HCC patients who received supportive care or chemotherapy as the initial treatment

\begin{tabular}{lcccc} 
Aetiology & HBV & HCV & HBV+HCV & NBNC \\
\hline Median survival (months) & 2.5 & 3.4 & 3.4 & 2.6 \\
1 year & $12.4 \%$ & $21.7 \%$ & $10.8 \%$ & $11.2 \%$ \\
3 year & $3.4 \%$ & $8.5 \%$ & $3.1 \%$ & $3.1 \%$ \\
5 year & $0.8 \%$ & $2.2 \%$ & $1.5 \%$ & $1.0 \%$ \\
10 year & $0.5 \%$ & $1.1 \%$ & $0 \%$ & $1.0 \%$ \\
\hline
\end{tabular}

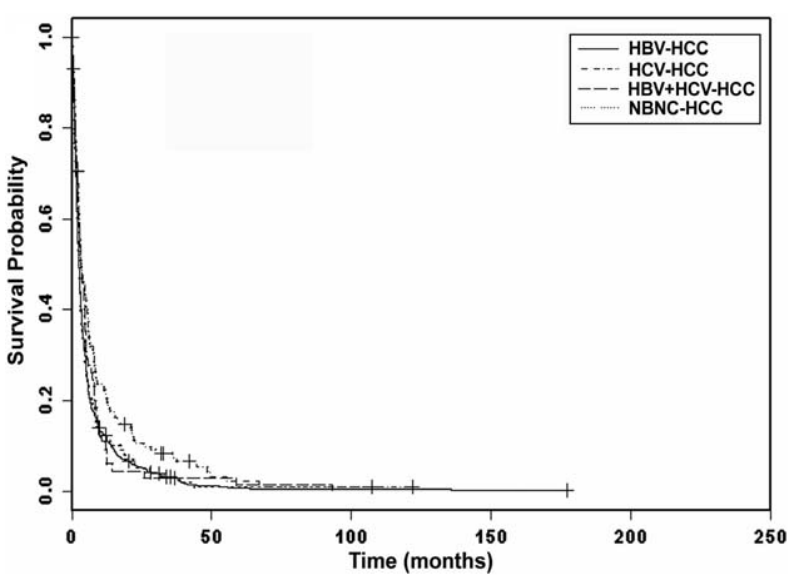

Fig. 5 - Kaplan-Meier estimated survival curves of HCC patients who underwent chemotherapy or supportive care. The HCV-HCC group showed a better survival than the HBVHCC $(p<0.001)$ or NBNC-HCC $(p<0.05)$ groups. HBV-HCC, HBV-related HCC; HCV-HCC, HCV-related HCC; HBV+HCVHCC, HBV plus HCV-related HCC, NBNC-HCC: non-HBV-nonHCV-related HCC.

chemotherapy as the initial treatment, the HCV-HCC patients had the best survival (Table 5, Fig. 5).

\section{Discussion}

Our data showed that HCV-HCC patients had the best overall survival. However, the better survival of HCV-HCC patients was found only in the non-surgical patients. This point was important because the treatment modality was a strong factor influencing survival. Our result was consistent with a recent observation that in the whole population, patients with HBV-HCC tended to have a poorer prognosis than those with HCV-HCC, and this difference became statistically significant among the patients with an advanced HCC. ${ }^{19}$ The previous conflicting results of comparing the prognosis of HBV-HCC versus HCV-HCC were probably due to the different inclusion criteria. Some investigators included only the surgical patients, ${ }^{4-6,8-10,13-15,17}$ while the others included all HCC patients. ${ }^{7,11,12,19,20}$

It was interesting to note that the better survival in the HCVHCC patients was not uniform over the course of time. Actually, the HCV-HCC patients had better prognosis in the initial 5 years. Then, the HCV-HCC patients lost their survival advantage at a longer follow-up period. The survival of HCV-HCC patients was worse than that in NBNC-HCC and HBV-HCC at 
10 years. A previous study did not find such paradox because the follow-up period was not long enough. The exact explanation for the paradox of survival advantage of HCV-HCC patients was not clear. One of the possibilities was that HCV-HCC, as compared with HBV-HCC, had a higher cumulated recurrence. ${ }^{21}$ An alternative explanation was that more HCV-HCC patients might die of non-liver-related disease since the HCVHCC patients were 12 years older than HBV-HCC patients.

The mean age of HCV-HCC patients was 12 years older than that of the HBV-HCC patients. The male/female ratio in HBV-HCC was 7.8, which was much higher than that found in the HCV-HCC group (1.7). These findings were the same as were reported in our previous multi-centre study. ${ }^{22}$ The lower WBC and platelet counts, and lower serum albumin levels in HCV-HCC patients might reflect the underlying higher percentage of liver cirrhosis in HCV-HCCs. However, the mean total bilirubin levels, the prolonged prothrombin time, and presence of ascites or encephalopathy, and Child-Pugh scores were similar among all four groups.

The mean tumour size was largest in the NBNC-HCC group. This was probably due to fewer NBNC-HCC patients receiving regular follow-up for the liver diseases since the two major risk factors for HCC, HBsAg and anti-HCV, were negative. The hepatic tumours might be detected only after symptoms occurred in the NBNC-HCC patients. Thus, the mean tumour size was larger. Though we defined HBsAg-negative, anti-HCV-negative HCC as NBNC-HCC, previous studies have demonstrated that some of these NBNC-HCC are caused by an occult HBV infection. ${ }^{23,24}$ Alcoholism may be one of the aetiologies of the NBNC-HCC. ${ }^{25}$ However, because this was a retrospective study, it was difficult to verify alcoholism among the NBNC-HCC patients. It was also interesting to note that the mean AFP levels were higher in the HBV-HCC groups than those in other groups. The higher AFP level in HBV-HCC might not be directly related to tumour size since the mean tumour size was larger in the NBNC-HCC group while the highest mean AFP level was in the HBV-HCC group, not in the NBNC-HCC group.

\section{Conclusion}

In conclusion, we found that HBV-HCC patients were characterised by younger ages, higher male/female ratio, higher serum AFP levels and larger tumour sizes. HCV-HCC patients were characterised by older ages, lower male/female ratio, lower WBC and platelet counts, lower serum albumin levels, higher percentage of liver cirrhosis, smaller tumour sizes. The HCV-HCC patients had best overall survival. However, the better survival in HCV-HCC patients was found only in the non-surgical patients and in the initial 5 years.

\section{Conflict of interest statement}

None declared.

\section{Acknowledgements}

This study was financially supported by the grants from the Department of Health, Taiwan (DOH90-HP-1002), the National
Health Research Institute, Taiwan (NHRI-EX94-9204PP), and the Liver Disease Prevention \& Treatment Research Foundation, Taiwan. We are indebted to our colleagues at the Cancer Registry, Office of Medical Record, NTUH, for their excellent work in the cancer registry system and to the physicians for their care of the patients.

\section{R E F E R E N C E S}

1. Chen CH, Chen DS. Hepatocellular carcinoma: 30 years' experience in Taiwan. J Formos Med Assoc 1992;91(Suppl. 3): S187-202.

2. Taiwan Cancer Registration System. <http:// crs.cph.ntu.edu.tw/>.

3. Chen CJ, Yu MW, Liaw YF. Epidemiological characteristics and risk factors of hepatocellular carcinoma. J Gastroenterol Hepatol 1997;12:S294-308.

4. Takenaka K, Yamamoto K, Taketomi A, et al. A comparison of the surgical results in patients with hepatitis $B$ versus hepatitis C-related hepatocellular carcinoma. Hepatology 1995;22:20-4.

5. Miyagawa S, Kawasaki S, Makuuchi M. Comparison of the characteristics of hepatocellular carcinoma between hepatitis $B$ and $C$ viral infection: tumor multicentricity in cirrhotic liver with hepatitis C. Hepatology 1996;24:307-10.

6. Yamanaka N, Tanaka T, Tanaka W, et al. Correlation of hepatitis virus serologic status with clinicopathologic features in patients undergoing hepatectomy for hepatocellular carcinoma. Cancer 1997;79:1509-15.

7. Tanizaki H, Ryu M, Kinoshita $\mathrm{T}$, et al. Comparison of clinical features and survival in patients with hepatitis B and C virusrelated hepatocellular carcinoma. Jpn J Clin Oncol 1997;27:67-70.

8. Shuto T, Hirohashi K, Kubo S, et al. Differences of resected hepatocellular carcinoma with hepatitis B or C virus. Hepatogastroenterology 1998;45:1722-5.

9. Chen MF, Jeng LB, Lee WC, Chen TC. Surgical results in patients with dual hepatitis B- and C-related hepatocellular carcinoma compared with hepatitis B- or C-related hepatocellular carcinoma. Surgery 1998;123:554-9.

10. Wu CC, Tang JS, Lin MC, Yeh DC, Liu TJ, P'Eng F K. Comparison of liver resection for hepatocellular carcinoma in hepatitis $\mathrm{B}$ and hepatitis C-related cirrhotic patients. Hepatogastroenterology 1999;46:651-5.

11. Tanabe G, Nuruki K, Baba Y, et al. A comparison of hepatocellular carcinoma associated with HBV or HCV infection. Hepatogastroenterology 1999;46:2442-6.

12. Dohmen $\mathrm{K}$, Shigematsu H, Irie K, Ishibashi H. Comparison of the clinical characteristics among hepatocellular carcinoma of hepatitis $B$, hepatitis $C$ and non-B non-C patients. Hepatogastroenterology 2003;50:2022-7.

13. Roayaie S, Haim MB, Emre S, et al. Comparison of surgical outcomes for hepatocellular carcinoma in patients with hepatitis B versus hepatitis C: a western experience. Ann Surg Oncol 2000;7:764-70.

14. Lee WC, Jeng LB, Chen MF. Hepatectomy for hepatitis B-, hepatitis C-, and dual hepatitis B- and C-related hepatocellular carcinoma in Taiwan. J Hepatobiliary Pancreat Surg 2000;7:265-9.

15. Chen TH, Tseng LM, Chau GY, et al. Clinicopathologic and prognostic differences between patients with hepatitis B- and C-related resectable hepatocellular carcinoma. J Formos Med Assoc 2001;100:443-8.

16. Benvegnu L, Alberti A. Patterns of hepatocellular carcinoma development in hepatitis B virus and hepatitis $C$ virus related cirrhosis. Antiviral Res 2001;52:199-207. 
17. Messerini L, Novelli L, Comin CE. Microvessel density and clinicopathological characteristics in hepatitis $C$ virus and hepatitis B virus related hepatocellular carcinoma. J Clin Pathol 2004;57:867-71.

18. Hsieh GY, Chen PC, Wang JD. Verification and correction of error for death registration data of the Department of Health R.O.C. between 1980 and 1997. Taiwan J Public Health 2002;21:329-38.

19. Cantarini MC, Trevisani F, Morselli-Labate AM, et al. Effect of the etiology of viral cirrhosis on the survival of patients with hepatocellular carcinoma. Am J Gastroenterol 2006;101:91-8.

20. Shiratori Y, Shiina S, Imamura $M$, et al. Characteristic difference of hepatocellular carcinoma between hepatitis B- and C- viral infection in Japan. Hepatology 1995;22:1027-33.
21. Huang $\mathrm{YH}, \mathrm{Wu} \mathrm{JC}$, Chen $\mathrm{CH}$, et al. Comparison of recurrence after hepatic resection in patients with hepatitis $\mathrm{B}$ vs. hepatitis C-related small hepatocellular carcinoma in hepatitis B virus endemic area. Liver Int 2005;25:236-41.

22. Lu SN, Su WW, Yang SS, et al. Secular trends and geographic variations of hepatitis B virus- and hepatitis $C$ virusassociated hepatocellular carcinoma in Taiwan. Int $J$ Cancer [in press].

23. Sheu JC, Huang GT, Shih LN, et al. Hepatitis C and B viruses in hepatitis B surface antigen-negative hepatocellular carcinoma. Gastroenterology 1992;103:1322-7.

24. Pollicino T, Squadrito G, Cerenzia G, et al. Hepatitis B virus maintains its pro-oncogenic properties in the case of occult HBV infection. Gastroenterology 2004;126:102-10.

25. Bruix J, Sherman M. Management of hepatocellular carcinoma. Hepatology 2005;42:1208-36. 